\title{
THE USE OF THE HILBERT HUANG TRANSFORMATION IN THE TESTING OF RAILWAY STRUCTURES
}

\author{
Jaroslav Smutný, Dušan Janoštík, Viktor Nohál \\ Brno University of Technology, Faculty of Civil Engineering \\ Institute of Railway Structures and Constructions, Veveri 95, 60200 Brno
}

\begin{abstract}
The aim of the paper is to introduce a less used method for the evaluation of non-stationary and especially transient phenomena in railway structures to the wider professional public. This method may find wide application in many technical and other fields. It is the so-called Hilbert-Huang transform. In this paper, its application in the study of dynamic phenomena occurring in a selected superstructure structure is shown. The calculation procedure of the presented transform differs from traditional tools, which include, for example, the short-term Fourier transform or the Wavelet transform. The paper includes a mathematical analysis and description of this transformation. Furthermore, the paper contains a description of the measurement method used, a discussion of the results obtained and recommendations for practice.
\end{abstract}

Keywords: Vibration, vibration acceleration, railway superstructure, analysis in the time and frequency domain, time-frequency methods

DOI: 10.36336/akustika20214029

\section{INTRODUCTION}

Today's rail transport is developing mainly in two basic areas. The first is high-speed rail transport, the second is rail transport in large urban agglomerations. The development of both areas brings new challenges. It should be pointed out that the increase in transport speeds and the operational load on the lines is causing new problems in the construction of the rail carriageway, irregular settlement of the rail bed, defects in the position of the long switch sleepers, loose spaces under the ends of the sleepers, crushing of the aggregate of the rail bed, defects in the running surface of the rail (waviness and slip waves) and increased levels of vibration and noise spreading to the surroundings of the lines. For urban rail transport, noise and vibration propagation problems are particularly addressed. New technical solutions are being implemented to address these problems. This applies in particular to different types of switch structures, different rail fastenings, different types of pads, sleepers, anti-vibration, and anti-noise measures, etc. The fastening of rails to sleepers, for example, is also a very important part of the superstructure.

In addition to theoretical analysis (simulation and modelling), the basis for the selection, an analysis and comparison of new parts of the superstructure is also experimental analysis carried out in the laboratory and on the track. An important part of the experiment includes methods for evaluating the measured data. These include signal processing in the time, frequency, and time-frequency domains. It is important to note that in the problem at hand, we mostly encounter transient and non-stationary signals. To evaluate them, both proven and less used techniques need to be used. In the following, a powerful method of time-frequency analysis is presented, which is hardly used in the field of railway structures evaluation. This is a pity, because this transformation can fully replace the currently commonly used methods or provide a new and more detailed view of the characteristics of the tested structures.

\section{THE BRIEF INTRODUCTION TO SIGNAL ANALYSIS METHODS}

Signal analysis is mainly performed in the time and frequency domain [1]. In the time domain, the time course of the measured variable is analyzed, or damping analysis in time, the effective value is calculated, etc. In the frequency domain, frequency spectra are calculated mainly by the Fourier transform method [2]. This conventional frequency analysis procedure cannot be used to determine the position of the frequency components in time. One possible way to analyze the temporal occurrence of frequency components, especially for transients and non-stationary signals is the use of so-called time-frequency transforms [3]. These can be divided according to the computational procedure into linear and nonlinear. In technical practice, the application of linear methods is predominant. The basic principle of calculation of all linear time-frequency procedures is the decomposition of the analyzed signal into elementary time-frequency units. Thus, the signal can be represented in the time-frequency plane as an $\mathrm{N}$-frequency sum according to the equation

$$
x(t)=\sum_{k=0}^{N-1} a_{k} \cdot \varphi_{k}(t),
$$

where

$\boldsymbol{x}(\boldsymbol{t})$ is the signal,

$\boldsymbol{\varphi}_{k}(\boldsymbol{t})$ are the time-frequency units (basis functions) and $\boldsymbol{a}_{\boldsymbol{k}}$ are the corresponding coefficients. The time-frequency position of these basis functions and the amplitude of their coefficients describe the signal in the time-frequency plane. 
Among the most widely used time-frequency procedures is the use of some modification of the Fourier transform. This is called Short Time Fourier Transform (STFT) according to the calculation procedure. The basic principle of this method is to divide the signal into sufficiently small realizations for which sufficient stationarity can be assumed. This is done by multiplying a certain window function and the signal. A Fourier transform is performed on each such slice. The Fourier transform, as is well known, decomposes the signal into a set of sine and cosine functions of different periods and amplitudes. The window is progressively shifted in time. Its defining integral is [4]

$$
\operatorname{STFT}(\tau, f)=\int_{-\infty}^{\infty}\left[x(t) \cdot g^{*}(t-\tau)\right] \cdot e^{-j \cdot 2 \cdot \pi \cdot f \cdot t} \cdot d t,
$$

where

g is the window function,

'*' is the complex conjunction,

$t \quad$ is the time,

$\tau$ is the time offset of the window,

$\boldsymbol{x}(\boldsymbol{t}) \quad$ is the temporal representation of the signal and

$\operatorname{STFT}(\boldsymbol{\tau}, \boldsymbol{f})$ is its time-frequency representation. Note that STFT localizes the frequency components in time with constant resolution.

Thus, it is not possible to provide excellent temporal and frequency resolution simultaneously. A wide time window gives a worse temporal resolution $\Delta \boldsymbol{t}$ but a relatively good frequency resolution $\Delta \omega \boldsymbol{\omega}$. A large bandwidth gives worse frequency resolution but relatively good temporal resolution. Some uncertainty is unavoidable.

The Wavelet Transform (WT) also belongs to the group of linear time-frequency procedures. In this transform, the signal is decomposed by a set of certain functions (called wavelets) The basic function of the transform is therefore the so-called mother wavelet. Other functions are derived from the mother wavelet by gradually expanding and shifting it in time. The continuous wavelet transform is defined by the integral equation [4]

$$
\begin{aligned}
& W T(\tau, s)=\int_{-\infty}^{\infty} x(t) \cdot \psi_{\tau, s}{ }^{*}(t) \cdot d t \\
& \psi_{\tau, s}(t)=\frac{1}{\sqrt{|s|}} \cdot \Psi\left(\frac{t-\tau}{s}\right),
\end{aligned}
$$

where

$\boldsymbol{t}$ is the time offset,

$\boldsymbol{s}$ is the scale (it is the inverse of the frequency)

$\boldsymbol{\Psi}$ is the transformation function, which is called the fundamental or mother wavelet,

$\boldsymbol{x}(\boldsymbol{t})$ is the signal being analysed, and

$\boldsymbol{W T}(\boldsymbol{t}, \boldsymbol{s})$ are the coefficients of the wavelet transformation.

There are several fundamental wavelets, one of the most widely used is the Morlet. It is defined as the product of a Fourier basis and a Gaussian probability function according to the relation [5]

$$
\psi(t)=e^{-j \cdot \omega_{0} \cdot t} \cdot e^{-\frac{t^{2}}{2}}
$$

The wavelet transform provides a so-called multi-resolution signal analysis, which is performed by applying a progressively expanded window function (parent wavelet). A narrow window is used for the analysis of high frequencies, and conversely a wide window is used for the analysis of low frequencies. For low frequencies, the height of the window is shorter, and the width is longer. This corresponds to better frequency and poorer temporal resolution. At high frequencies, the width is reduced, and the height is increased. This change in window size means better temporal and worse frequency resolution.

Thus, the advantage of both linear transforms is mainly the speed of computation and satisfactory time-frequency resolution. Their main disadvantage is that the resulting time-frequency resolution is limited by the so-called Heisenberg uncertainty principle [5]:

$$
\Delta f \cdot \Delta t \approx \mathrm{konst}
$$

Therefore, the signal component cannot be represented as a point in time-frequency space. Therefore, it is only possible to determine its position within the rectangle $\boldsymbol{\Delta t} \cdot \boldsymbol{\Delta} \boldsymbol{f}$ in each time-frequency domain ( $\Delta t$ represents the minimum time interval - time step, $\boldsymbol{\Delta} \boldsymbol{f}$ represents the minimum frequency interval - frequency step). This fact can affect both time and frequency resolution, and therefore the analysis of the temporal occurrence of important frequency components.

\section{DESCRIPTION OF THE HILBERT HUANG TRANSFORMATION}

One of the distinguishing features of the Hilbert Huang transform is that it decomposes the signal under analysis in a different way than other linear time-frequency procedures. As a result, the resulting resolution of this transform in time and frequency is not limited by the so-called Heisenberg uncertainty principle. This fact implies a high resolution in both time and frequency. This is manifested by the "very precise localization of the prominent frequency components in time. Which represents the biggest advantage of this transformation over other procedures.

To begin with, it should be noted that the classical methods use the classical approach of frequency decomposition based on a certain repetition of the process, i.e. periodicity.

In signal analysis theory there is yet another possibility. The instantaneous frequency can be determined on the basis of the existence of the so-called analytical signal. An analytical signal is an artificially created signal from an initial signal such as the measured signal. An imaginary part is attached to the real signal in the definition of an analytical signal. The analytical signal is therefore a complex function of time. Its time course can be represented in algebraic complex number form, i.e. separately real and imaginary parts, or in exponential form, i.e. separately absolute value and phase. The analytical signal can be described by equation $7[6,7]$.

$$
z(t)=s(t)+j \hat{s}(t)
$$


The Hilbert transform is used to obtain the imaginary component of the real signal. For any time signal $\boldsymbol{s}(\boldsymbol{t})$, the Hilbert transform of this signal is defined by [8]

$$
H[s(t)]=\hat{s}(t)=\frac{j}{\pi} P \int_{-\infty}^{\infty} \frac{s(\tau)}{t-\tau} d \tau
$$

where

$\boldsymbol{s}(\boldsymbol{t})$ is the real signal,

$\boldsymbol{j}$ is an imaginary unit, $\boldsymbol{t}$,

$\boldsymbol{\tau}$ time,

$\boldsymbol{P}$ is the principal Cauchy value,

$\hat{\boldsymbol{s}}(\boldsymbol{t})$ represents the transformed signal.

The instantaneous frequency can be expressed by the following equation [9]

$$
f_{i}=\frac{1}{2 \pi} \cdot \frac{d \varphi(t)}{d t}
$$

where

$\varphi(t)$ represents the instantaneous phase of the signal.

Equation (9) represents the derivative of the phase, which takes on exactly one value at each point in time $\boldsymbol{t}$. Real signals are usually made up of a wide range of frequencies. The calculation can then lead to negative values of frequencies, which is very difficult to interpret physically. Furthermore, it is not possible to determine when which frequency component originated and when it was terminated in the process. Thus, it is not possible to directly calculate the instantaneous frequency from the real signal. However, around the year 2000, scientist E. Huang published a procedure by which it is possible to decompose a real signal into individual components so that the instantaneous frequency can be calculated for each of them. The whole approach is now known as Hilbert-Huang transform [9].

Thus, the Hilbert-Huang transform represents a lesser known and lesser used method for the analysis of nonlinear, transient and non-stationary signals. Briefly, its calculation can be divided into two parts. The first part is the so-called empirical modal decomposition and the second part involves the construction of the so-called Hilbert spectrum, which is essentially the energy distribution in the time-frequency domain using the Hilbert transform. The empirical modal decomposition decomposes the analyzed signal into its own modal functions, which are based on the local properties of the signal $[10,11]$. This fact allows a physically "meaningful" definition of the instantaneous frequency, and thus removes the need to use often less appropriate harmonic functions to represent nonlinear and nonstationary signals. The term empirical modal decomposition can be thought of as a method used to decompose any signal $\boldsymbol{s}(\boldsymbol{t})$ into a finite number of modal functions. The modal functions must essentially satisfy two basic conditions [11, 12]. The first involves the symmetry of the envelopes formed by the local minima and maxima with respect to the zero midpoint, and the second involves the assumption that the number of local extrema is equal to the number of intersections with the time axis. One possible procedure for computing the empirical modal decomposition is described below. Its implementation proceeds in several steps.

The first step consists in finding local extrema (maxima and minima) in the given signal $\boldsymbol{s}(\boldsymbol{t})$. These extrema are then interpolated with a suitable curve, for example a cubic spline. This produces two envelopes from the original signal - the upper $\boldsymbol{e}_{\text {max }}(t)$ and the lower $\boldsymbol{e}_{\text {min }}(t)$. The midpoint of the two envelopes is then calculated. Calculate the difference between the signal and the midpoint. In theory, this difference could represent the first component. If the calculation does not satisfy the requirements for the modal function, which is very likely due to the spline approximation, we need to continue iterating. Note that due to the spline approximation, new extremes, i.e., overshoots or undershoots in the envelope, may arise. The computation procedure must be repeated until the resulting component satisfies the specified conditions. It is worth noting here that the iterative process essentially filters out the low-frequency components and smooths out the amplitude differences.

The value of the standard deviation, which is calculated from two consecutive results of the iteration process, can be very well used as a criterion. E. Huang has set the typical value of the standard deviation for the termination of an iteration in the interval between 0.2 and 0.3 . Note that the first modal component represents the highest frequency component of the signal $\boldsymbol{s}(\boldsymbol{t})$. If we separate the computed component from the signal, we obtain the so-called residual. The residual contains components with lower frequencies. It can be marked as a new signal and the procedure can be repeated. The original signal can therefore be described by the following equation [12]

$$
s(t)=\sum_{i=1}^{n} c_{i}+r_{n}
$$

where

$\boldsymbol{s}(\boldsymbol{t})$ is the original signal,

$\boldsymbol{r}_{n}$ is the residual, and

$c_{i}$ represents the component.

Note that the decomposition can be terminated based on a predetermined number of iterations or when the residual becomes a monotonic function from which no further modal functions can be separated. Once we have calculated the individual components of the decompositions described above, we can apply the Hilbert transform to each component and calculate the instantaneous frequency. This procedure can be expressed by the following equation $[13,14]$.

$$
s(t)=\sum_{k=1}^{n} a_{k}(t) \cdot e^{j \cdot \int \omega_{k}(t) \cdot d t} .
$$

Thus, equation (5) expresses the instantaneous amplitude $\boldsymbol{a}_{\boldsymbol{k}}(\boldsymbol{t})$ and the instantaneous frequency $\boldsymbol{\omega}(\boldsymbol{t})$ as a function of time, while also allowing the amplitude to be expressed as a function of frequency and time, and to output this into a 3-dimensional graph. This is then the Hilbert amplitude spectrum, which expresses the energy distribution in the time-frequency domain.

From the above description, it is clear that the Hilbert-Huang transform represents a certain alternative to, for example, the Fourier transform or the Wavelet transform, or other time-frequency methods [15], with the difference that it decomposes the measured signal in a different way. That is, not into predefined functions such as trigonometric functions, mother wavelet [16], etc., but into functions defined on the basis of the signal under investigation. It then transforms the original measured signal into an analytical signal from which the instantaneous signal characteristics (instantaneous frequency, amplitude and phase) can be obtained. These parameters can be of great interest for the detection of real dynamic processes [15]. 
It can be reasonably assumed that the outputs of the Hilbert-Huang transform can also be very well combined with selected machine classification methods [16]. This fact is evident from many applications in both engineering and science fields.

\section{DESCRIPTION OF MEASUREMENT AND EVALUATION METHODS}

The measurements were carried out on a test sample. This specimen consisted of the head of a concrete sleeper B91S on which the 60 E1 rail specimen was mounted using the Vossloh E14 rail fastening (Fig. 1). It should be noted that this is a baseless fastening, which is classified as a fastening with increased vertical flexibility. The use of E14 is suitable where increased damping of dynamic effects is required. The design of this fastening is based in principle on the Type 300 fastening for fixed railway track. The highly elastic Zwp E14 NT/150 pad partially replaces the damping effects of the track bed, so that the $\mathrm{E} 14$ fastening can be used to advantage, for example, in locations where it is not possible to provide a track bed of sufficient thickness.

The sample was placed in an artificially created track bed during the measurements. It should be noted that the dynamic and acoustic parameters of all fastenings used in the Czech Republic are investigated at the authors' workplace. The measured values are converted into parameters which are stored in an expert database and used for various purposes. These are mainly for later comparisons, further analyses and as inputs to mathematical models in the final theses of students. This expert database is implemented using the services of the MySQL software system on the institute's NAS storage. Both the measurement data and the calculated parameters are stored here.

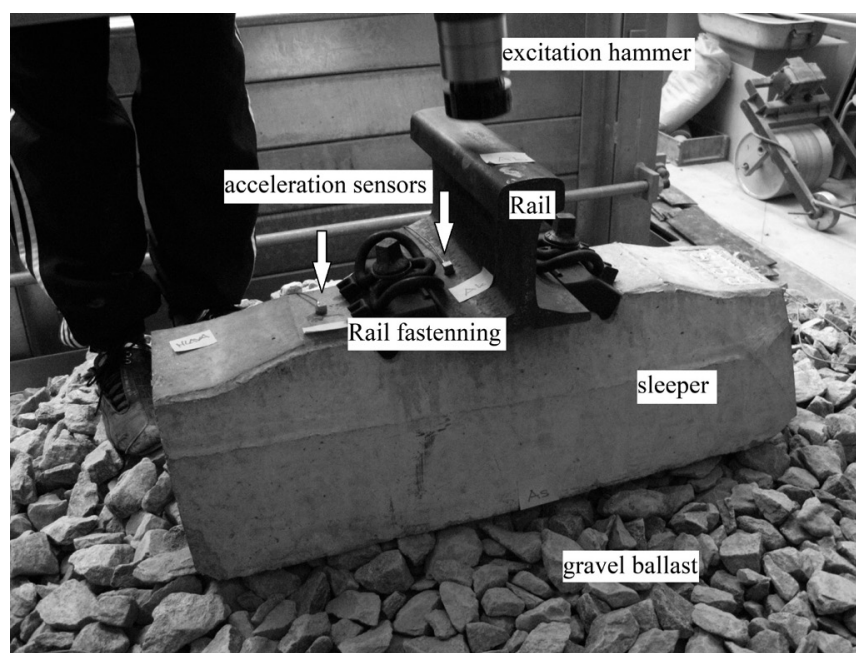

Fig. 1: The view of the Vossloh E14 rail fastening sample

For the experimental investigation of the dynamic properties of the assembled test specimen, a method based on the measurement of the response to mechanical impact [17] was used. The mechanical impact was induced by a special hammer, which has an embedded force transducer, in the radial direction on the rail head. The response was measured by acceleration sensors at the rail foot, sleeper and in the gravel bed. Impulse transfer functions were calculated from the response signals due to normalization to the excitation signal. In this way, a normalized time history of the acceleration oscillation was obtained.

This measuring method including instrumentation is described in detail in the literature [18]. Time domain, frequency domain and time-frequency domain analysis were used to evaluate the measured data. The Multi-taper method [19] was proposed for the analysis in the frequency domain. The previously used time-frequency characteristics (Wavelet Transform, Short Time Fourier Transform and so on) were supplemented with the outputs from the Hilbert-Huang transform.

\section{MEASUREMENT ASSESSMENT}

The evaluation software was developed in Python. This was because commercial software commonly used for similar analyses (e.g. Dewesoft from Dewetron company, Pulse and BK Connect from Bruel\&Kjaer company, Flexpro from Weisang company, LabView from National Instruments company , etc.) did not include this transformation.

Following the literature [19], the outputs of the analyses were again organized into a triplet of graphs (Fig. 2, Fig 3). Note that this group of graphs probably provides the best opportunity for more detailed analyses as well.

The top graph of each figure shows the impulse response function waveform. The left graph shows the amplitude spectrum of the vibrational response. The middle graph shows a $3 \mathrm{D}$ representation of the time-frequency waveform of the amplitude spectrum of the vibration response.

The values of the spectra are expressed in decibel scale by different colours (or grayscale). Note that the maximum value is the colour black. Again, it should be mentioned that for a more detailed insight into the dynamic characteristics, time and frequency slices can be displayed within the middle gra$\mathrm{ph}$. In the former case, these are plots providing individual frequency spectra at different times, while in the latter case; they are time histories of selected frequency components.

The upper plot of Fig. 2 shows the impulse response function obtained from the accelerometer sensor that was placed at the foot of the rail. This waveform shows the extremes, waveform and decay time. The left plot of Fig. 2 shows the amplitude spectrum.

Several prominent frequencies can be seen in the plot $(300 \mathrm{~Hz}, 670 \mathrm{~Hz}, 730 \mathrm{~Hz}, 760 \mathrm{~Hz}, 1050 \mathrm{~Hz}$ and $1150 \mathrm{~Hz}$ ). In addition, there is a relatively broad frequency cluster with a peak at $590 \mathrm{~Hz}$. The time-frequency amplitude spectrum calculated by applying the Hilbert-Huang transform to the impulse response function is shown in the middle plot of Fig. 2.

As can be seen from this plot, the same frequency components are evident as from the amplitude spectrum plot. However, their temporal occurrence differs significantly. The $300 \mathrm{~Hz}$ frequency component is the highest and for a relatively long time (relative to the other frequency components). Other components that appear in the signal for a relatively long time include $1050 \mathrm{~Hz}, 760 \mathrm{~Hz}$ and others.

The waveform of the impulse response function obtained from the accelerometer sensor placed on the concrete sleeper has a different character. From the upper graph of figure Fig. 3 shows that the maximum and minimum take lower values than in figure Fig. 2. This is due to the effect of the wave progression over the obstacle in the form of the rail attachment, the pad under the rail and the sleeper. The course of the amplitude spectrum (left plot of Fig. 3 ) also differs from 
the characteristics calculated for the sensor located at the foot of the rail. The most significant components detected from the sensor on the rail also appear in the spectrum, but take on much lower values.

The same conclusions are provided by the middle plot of Fig. 3, which presents a time-frequency representation of the Hilbert-Huang transform coefficients. The longest-occurring component of the spectrum is the $300 \mathrm{~Hz}$ frequency. Its temporal occurrence is about half that of the on-rail sensor. This fact also applies to other significant components of the spectrum. It can therefore be concluded that the vibration waves are significantly damped due to the mounting. This fact should also result in a significant reduction of dynamic and acoustic events even in normal operation [19].

\section{CONCLUSIONS}

Since the publication of this progressive method around 2000, several further modifications have been developed. Due to its nature, empirical modal decomposition finds application in a wide range of applications. Let us recall, for example, the removal of noise in the measured signal, the visualization of various sources and mechanisms, the analysis of image data, etc. It will certainly find its use also in the analysis of acoustic and vibrational processes.

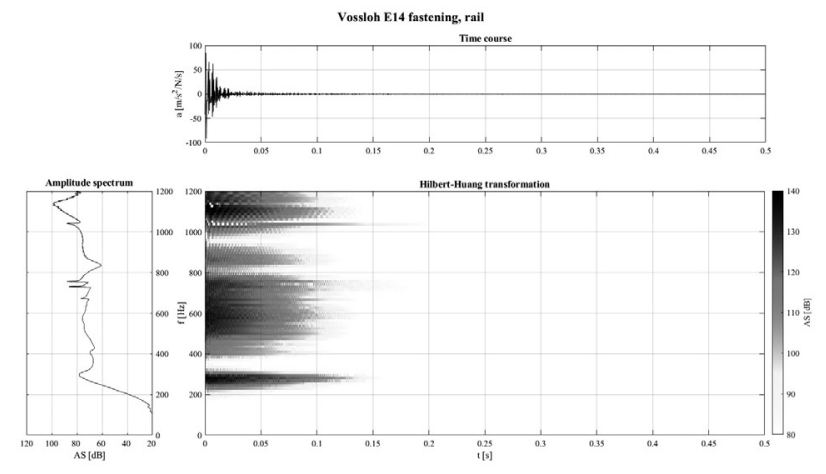

Fig. 2: The time, frequency and time-frequency analysis of the dynamic response on rail, Vossloh 1414 rail fastening

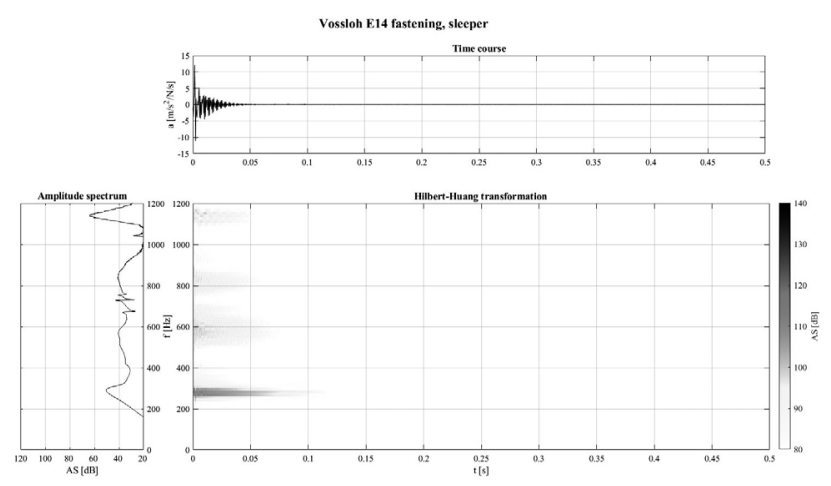

Fig. 3: The time, frequency and time-frequency analysis of the dynamic response on sleeper, Vossloh E14 rail fastening

The Hilbert Huang transform processes the measured signal in a different way than other linear time-frequency transforms. Note that it does not decompose the signal into predefined functions such as trigonometric functions, mother wavelet, etc., but into functions defined based on the signal under in- vestigation. It then transforms the original signal into an analytical signal from which the instantaneous properties of the signal (instantaneous frequency, amplitude, and phase) can be obtained. These parameters are very important for the detection of real dynamic events. This fact is particularly beneficial when extracting frequency components from non-linear and strongly non-stationary signals. Another great advantage of this transformation is that the calculated outputs are not affected by the so-called Heisenberg uncertainty principle. This fact involves the accurate localization of the significant frequency components contained in the measured signal. A certain disadvantage may be the complexity of the calculation. This could be an obstacle in the implementation of this method in measuring systems based on single-chip computers and microcontrollers, but not in conventional computer technology.

The paper presents the theoretical analysis and practical application of the Hilbert-Huang transform for the evaluation of dynamic phenomena occurring in a selected superstructure structure. On the basis of the measurements and analyses carried out, it is possible to express the opinion that the Hilbert-Huang transform can also be used as an additional supporting method in selected railway engineering applications, especially in the evaluation of short-term dynamic effects on the railway structure from passing trains. It should be noted that the problem focus will certainly be directed to the area of detection of harmful phenomena in the track (deteriorating condition of the track superstructure due to rail defects, defects in the area of fasteners, sleepers, or track bed), but also to the area of train set condition assessment. The presented transformation seems to be a very interesting approach especially for the assessment of non-stationary and transient processes.

Also, the authors of this paper anticipate the use of two machine classification methods, namely the support vector method and the neural network, in the future process of railway structure evaluation. In both cases, it will be a machine learning method with a teacher.

The authors recommend continuing the ongoing work, to incorporate the presented transformation into existing methodologies for the evaluation of dynamic effects on railway structures, both under laboratory conditions and in-situ conditions.

\section{ACKNOWLEDGEMENTS}

This paper was supported by the project FAST-S-21-7401, "Evaluation of dynamic effects acting on a structure by advanced mathematical analysis methods". 


\section{REFERENCES}

[1] Smutny J., Pazdera L.: New techniques in analysis of dynamic parameters rail fastening, INSIGHT, ISSN 1354-2575, InSight, 2004.

[2] Champeney D. C.: A Handbook of Fourier Theorems, Cambridge University Press, 1987.

[3] Smutny J.: Measurement and Analysis of Dynamic and Acoustic Parameters of Rail Fastening, NDT \& E International - Independent Nondestructive Testing and Evaluation, ISSN 0963-8695, Elsevier, 2004.

[4] Flandrin P.: Time-frequency/Time-scale analysis, Academic Press, 1999.

[5] Putra T., Abdullah S., Nuawi M.Z., Yunoh M.F.M.: The Morlet and Daubechies wavelet transforms for fatigue strain signal analysis, Applied Mechanics and Materials, 2018, Vol. 471, pp. 197-202.

[6] Huang N.E., Shen Z., Long S.R., Wu M.C., Shih H.H., Zheng Q., Yen N. C., Tung C.C., Liu H-H.: The empirical mode decomposition and the Hilbert spectrum for nonlinear and non-stationary time series analysis, Proc. R. Soc. Lond. A 454 (1998), pp. $903-995$.

[7] Rilling G., Flandrin P., Goncalves P.: On empirical mode decomposition and its algorithms, Nonlinear Signal Processing, IEEE EURASIPWorkshop, Grado, Italy, 2003.

[8] Huang, N.E.: New method for nonlinear and nonstationary time series analysis: empirical mode decomposition and Hilbert spectral analysis", Proceedings of SPIE 4056, Wavelet Application VII, Orlando, FL, 24-28 April, 2000, pp. $197-210$.

[9] Huang N. E., Shen S. S. P.: Hilbert-Huang transform and its applications, World Scientfic Publishing, 2005, pp. 1-24.

[10] Torres M. E., Colominas M. A., Schlotthauer G. S., Flandrin P.: A Complete Ensemble Empirical Mode Decomposition with Adaptive Noise, IEEE international Conference on Acoustic, Speech and Signal Processign (ICASSP), May 2011, pp. 41444147.

[11] Junsheng C., Dejie Y. Yu Y.: Research on the intrinsic mode function (IMF) criterion in EMD method, Mechanical System and Signal Processing, 2004, Vol. 20 No. 4, pp. 817-824.

[12] Zhang R. R., Ma S., Safak E., Hartzell S.: Hilbert-Huang transform Analysis of Dynamic and Earthquake Motion recordings, Journal of Engineering Mechanics, vol. 129, August 2003, pp. 861-875.

[13] N. E. Huang, and Z. Wu, "A Review on Hilbert-Huang Transform Method and its Applications to Geophysical Studies," in Reviews of Geophysics, 46, 2008, pp. 1-23.

[14] N. E. Huang, X. Chen, M-T. Lo, and Z. Wu, "On Hilbert spectral representation: A true time-frequency representation for nonlinear and nonstationary data," in Advances in Adaptive Data Analysis, vol. 3, no. 1 and 2, 2011, pp.63-93.

[15] Chiang W. L., Chiou D. J., Chen C. W., Tang J. P., Hsu W. K., Liu, T. Y.: Detecting the sensitivity of structural damage based on the Hilbert-Huang transform approach, Engineering Computations, 2010, Vol. 27 No. 7, pp. 799-818.

[16] Xing Z., Qu J., Chai Y., Tang Q. Zhou Y.: Gear fault diagnosis under variable conditions with intrinsic time-scale decomposition and support vector machine, Journal of Mechanical Science and Technology, 2017, Vol. 31 No. 2, pp. 545-555.

[17] Smutny J., Janostik D., Pazdera L., Nohal V.: The use of Unterberger transformation on analysis of dynamic parameters of rail fastenings, Akustika, 2020, roč. 35, č. 1, s. 33-40, ISSN 1801-9064.

[18] Smutny J., Janostik D., Nohal V.: Application of unconventional methods for frequency analysis in acoustics, Akustika, 2020, roč. 36, č. 2, s. 26-33. ISSN 1801-9064.

[19] Smutny J., Pazdera L., Tomandl V.: Assessment of dynamic parameters of rail fastening. Communications, 2016, roč. 18, č. 3, s. 65-70. ISSN: 1335-4205. 


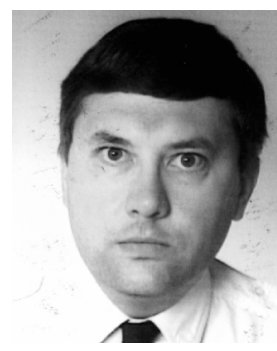

Jaroslav Smutný He graduated from the Faculty of Electrical Engineering, TU Brno, specialisation technology of radio and communication devices. He works as a researcher-teacher at the Department of railway constructions and structures. He was graduated Ph.D. at the specialisation Theory of structures (Ph.D. thesis Modern Method of Noise and Vibration Analysis Applied on Railway Structures). College lecturer - 2002 ("Time Frequency Analysis of Constructional Structures and Materials"). 2009- Professor - Constructions and Transport Structures. He is member of Czech association for geo-information, Czech acoustic society andTime frequency user club. Within the framework of the scientific and research and pedagogical activities he devotes his activities especially to the problems of geographical information systems, the experimental analysis of structures, the analysis of noise and vibrations, the problems of applying the methods of the artificial intelligence in experimental analysis of structures, the modelling of structures etc.

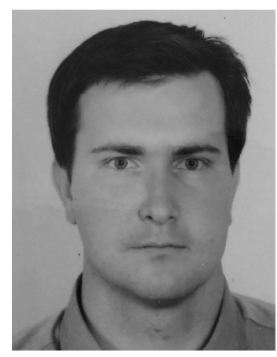

Dušan Janoštík was born on the 26nd august 1972 in Hranice na Moravě, Czech Republic. He graduated from his master degrees at the Faculty of Civil Engineering, Brno University of technology in 1998 and spe-cialization was railway construction. For a long time he worked in the field of automated data processing and computer technology. He was also a computer network administrator. Currently he is studying doctoral program Civil engineering. Within his studies he focuses on the problems of railway structures, geographic information systems, methods of data analysis by selected methods of artificial intelligence and wireless data transmission.

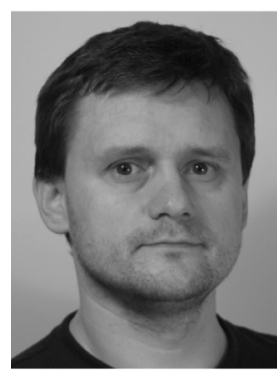

Viktor Nohál was born on the 23nd February 1979 in Zlin, the Czech Republic. He graduated from his master degree studies at the Faculty of Civil Engineering, Brno University of Technology in 2004 and his specialization was traffic construction. Currently he is studying doctoral program "Civil Engineering". At the same time he works in design and consulting company. He worked on projects from these areas - traffic engineering and land-use planning documentation, but he also has experience with design activities of highway construction. During his doctoral study he focuses on the measurement of physical values and on intelligent transport systems and their potential optimizations. 